\title{
UMA APLICAÇÃO MODELO DA ARBITRAGE PRICING THEORY PARA O SETOR DE BENS INDUSTRIAIS
}

\section{AN APPLICATION OF THE ARBITRAGE PRICING MODEL FOR THE INDUSTRIAL GOODS SECTOR}

\author{
Altamir Schembergue* \\ Emerson Martins Hilgemberg**
}

\begin{abstract}
RESUMO
Este artigo analisou a influência dos fatores macroeconômicos no retorno das ações do setor de bens industriais negociados na Bolsa de Valores de São Paulo (Bovespa) no período de dezembro de 1999 a junho de 2010. Para tanto foi utilizada a abordagem da Arbitrage Pricing Theory (APT), realizando-se regressões em séries temporais, as quais tinham como variáveis explicativas dos retornos o índice Bovespa, a produção industrial, a taxa de inflação, a balança comercial, a taxa Selic, o índice Dow Jones e taxa de câmbio. Estas variáveis mostraram-se significativas para todas as ações, porém observaram-se diferenças nos resultados por conta das características individuais de cada empresa (fatores idiossincráticos). O índice Bovespa foi significativo para oito das nove ações analisadas, a taxa de inflação foi significativa para cinco das nove ações e a variável taxa de câmbio não se mostrou significativa para nenhuma das ações.
\end{abstract}

Palavras- chave: APT. Precificação de ativos. Bens industriais,

\begin{abstract}
This article analyzed the influence of macroeconomic factors on the return of shares from the Industrial Goods sector traded on Sao Paulo Stock Exchange (Bovespa) from December 1999 to June 2010. The study used the Arbitrage Pricing Theory approach and performed time-series regressions whose explanatory variables were the Bovespa index, industrial production, inflation rate, trade balance, Selic interest rate, Dow Jones index and exchange rate. The variables were significant for all securities, however, there were differences in the results due to unique characteristics of individual firms (idiosyncratic factors). Bovespa index was significant for eight of the nine stocks analyzed, the inflation rate was significant for five of the nine stocks and exchange rate variable was not significant for any of the shares.
\end{abstract}

Keywords: APT. Asset pricing. Industrial goods sector.

\footnotetext{
"Mestrando em Economia Aplicada pela Universidade Federal de Viçosa. Email: aschembergue@bol.com.br

** Doutor em Economia Aplicada pela Universidade de São Paulo (USP) e mestrado em Economia Aplicada pela mesma instituição. Professor adjunto da Universidade Estadual de Ponta Grossa (UEPG). Email: ehilgemberg@uepg.br
} 


\section{Introdução}

O modelo Capital Asset Pricing Model (CAPM) foi considerado durante muito tempo como a forma mais segura para analisar os retornos dos ativos ou de um conjunto de ativos. Entretanto, embora esse modelo seja extremamente respeitado e útil, ele sofre diversas críticas, derivadas de seus pressupostos e restrições.

O modelo de apreçamento por arbitragem (Arbitrage Pricing Theory, ou simplesmente APT) foi desenvolvido por Ross (1976) a partir de algumas inconsistências demonstradas pelo CAPM. O modelo da APT pretende responder a questões que o CAPM não é capaz. Para tanto, abandona a hipótese do mercado como único fator determinante de risco dos ativos e considera outros fatores na explicação do processo gerador de retorno dos ativos. Os fatores utilizados pela APT podem ser tanto macroeconômicos quanto fatores estruturais relacionados ao setor de atuação das empresas.

Esse trabalho utilizará o modelo da APT para verificar se existe essa relação entre os retornos e os fatores macroeconômicos, especificamente num conjunto de ações de empresas pertencentes ao setor de bens industriais negociadas no mercado acionário brasileiro no período de dezembro de 1999 a junho de 2010.

O artigo está organizado em cinco seções, além desta introdução. A seção 2 trata da fundamentação teórica, a seção 3 traz a metodologia utilizada no estudo, a seção 4 analisa os resultados obtidos e, finalmente, a seção 5 trata das considerações finais.

\section{Fundamentação teórica}

No modelo CAPM, o retorno esperado de qualquer ativo $i$ é dado por:

$$
\overline{R_{i}}=R_{F}+\beta_{i}\left(\overline{R_{m}}-R_{F}\right)
$$

Onde: $\bar{R}_{i}$ é o retorno esperado do ativo $i ; R_{F}$ é a rentabilidade do ativo livre de risco ou renda fixa; $\bar{R}_{m}$ é a rentabilidade do ativo de mercado; $\beta_{I}$ é um parâmetro que mede o risco não sistemático do $i$ -ésimo ativo.

A equação (1) descreve o retorno esperado de todos os ativos e carteiras de ativos na economia.
Logo, o retorno esperado de qualquer ativo ou carteira, eficiente ou não, pode ser determinado com essa relação. Assim, para calcular o retorno esperado de qualquer ação é necessário anenas conhecer o prêmio de risco do mercado $\left(\overline{R_{m}}-R_{F}\right)$ e o beta da ação.

O modelo da APT desenvolvido por Ross (1976) é um modelo alternativo ao CAPM. A principal diferença está no fato de que ele não assume que o mercado seja a única fonte explicativa dos retornos. Ao contrário, o modelo APT assume a existência de um processo gerador de retornos, o qual incorpora uma série de fatores de risco responsáveis por retornos excedentes àqueles obtidos de um ativo sem risco.

Segundo Burmeister, Roll e Ross (2003), dado que os lucros vindos de arbitragem pura tendem a zero, não é necessário fazer suposições restritivas sobre preferências ou probabilidade de distribuições, fornecendo uma base lógica para compensação entre retorno esperado e risco.Ou seja, o retorno esperado de uma carteira composta por $K$ fatores poderia ser escrita da seguinte forma:

$$
E\left(R_{P}\right)=R_{F}+\beta_{1}\left(R_{1}-R_{F}\right)+\beta_{2}\left(R_{2}-R_{F}\right)+\cdots+\beta_{i}\left(R_{i}-R_{F}\right)+\sum_{i=1}^{n} \frac{1}{k} \varepsilon_{i}
$$

\section{Aspectos metodológicos}

Para estimar o retorno esperado, Ross et.al (1986) utilizaram a produção industrial, inflação, variação no prêmio risco e a diferença em termos de taxa de juros de longo e curto prazo como forma de substituição do fator único proposto pelo CAPM.

Callado et.al (2009) utilizaram a taxa de juros (SELIC), taxa de câmbio e a taxa de inflação (IPCA). Kude (1998) apud Rostango e Kloeckner (2004) identificou oito fatores como fonte de risco.

Como a APT não estabelece a priori quais as variáveis devem ser utilizadas, a inclusão ou exclusão de variáveis é um tanto arbitrária. Tudo que a teoria fornece é a ideia de que existem $k$ fatores que influenciam os retornos das ações.

Neste trabalho, portanto, foram consideradas as seguintes variáveis: taxa SELIC (Over), produção industrial, taxa de inflação (IPCA), balança comercial (saldo em US\$ milhões FOB), taxa de câmbio (PTAX), Ibovespa e índice Dow Jones. 
Os critérios de seleção das empresas foram os seguintes (CALLADO et al., 2009):

- As empresas devem ser negociadas na Bovespa;

- As empresas devem ter o Brasil como sede (país origem);

- As empresas devem possuir cotações mensais (preço de fechamento) ininterruptas no período entre dezembro de 1999 e junho de 2010.
O estabelecimento desses critérios assegurou a homogeneidade da amostra e proporcionou maior robustez aos resultados.

As empresas que compuseram a amostra estão listadas no quadro 1.

Os dados históricos relativos aos preços de fechamento das ações foram obtidos por intermédio do programa Grafix.

Quadro 1 - Empresas selecionadas

\begin{tabular}{|c|c|c|c|c|}
\hline \multirow{2}{*}{$\begin{array}{l}\text { SETOR } \\
\text { ECONÔMICO }\end{array}$} & \multirow{2}{*}{ SUBSETOR } & \multirow{2}{*}{ SEGMENTO } & \multicolumn{2}{|c|}{ LISTAGEM } \\
\hline & & & CÓDIGO & SEGMENTO \\
\hline \multirow{15}{*}{ Bens Industriais } & $\begin{array}{l}\text { Material de } \\
\text { Transporte }\end{array}$ & Material Aeronáutico & & \\
\hline & & EMBRAER & EMBR & NM \\
\hline & & Material Rodoviário & & \\
\hline & & RANDON PART & RAPT & N1 \\
\hline & & MARCOPOLO & POMO & N2 \\
\hline & & METAL LEVE & LEVE & \\
\hline & $\begin{array}{c}\text { Máquinas e } \\
\text { Equipamentos }\end{array}$ & Máqs. e Equips. Industriais & & \\
\hline & & BARDELLA & BDLL & \\
\hline & & INEPAR & INEP & \\
\hline & & Máqs. e Equips. Construção e Agrícolas & & \\
\hline & & METISA & MTSA & \\
\hline & & Armas e Munições & & \\
\hline & & FORJA TAURUS & FJTA & \\
\hline & Comércio & Material de Transporte & & \\
\hline & & WLM IND COM & SGAS & \\
\hline
\end{tabular}

Fonte: BM\&F Bovespa

Uma vez que o modelo APT não traz uma indicação ex ante das variáveis explicativas a serem introduzidas no modelo, efetuou-se uma análise de correlação entre as variáveis explicativas que seriam inicialmente utilizadas. $\mathrm{O}$ critério utilizado foi o de que, caso dois fatores econômicos (variáveis) tivessem correlação de 0,70 ou maior, um seria excluído; no entanto os testes não apontaram para a exclusão de nenhuma variável.

Segundo Gujarati (2006), para que seja possível estimar modelos de regressão em séries temporais, é necessário verificar se as séries analisadas são estacionárias, ou seja, se são integradas de ordem zero $I(0)$, ou integradas de mesma ordem $I(d)$, onde (d) representa o número de vezes que a série tem de ser diferenciada para que se torne estacionária.

A estacionariedade foi testada por meio do teste de Dickey- Fuller Aumentado (ADF) confor- me calculado pelo software Eviews 5. Os resultados mostraram que, dentre as variáveis analisadas, apenas a taxa Selic não era estacionária em nível. Porém, Gujarati (2006) afirma que, quando os erros ( $\left.\mu_{t}\right)$ da equação de regressão estimada são estacionários, os resultados obtidos não serão espúrios. Ou seja, a combinação das tendências estocásticas das séries se anulam entre si, de tal forma que sua combinação linear é $I(0)$. Desse modo, os resultados da regressão farão sentido, e nesse caso pode-se dizer que as séries são cointegradas, ou seja, as variáveis apresentam uma relação de longo prazo, ou de equilíbrio. 


\section{Análise dos resultados}

A regressão estimada foi a seguinte:

$$
R_{i}=\bar{R}_{i}+\beta_{1 i} \text { Ibv }+\beta_{2 i} \text { DJones }+\beta_{3 i} \text { Selic }+\beta_{4 i} \text { PIM }+\beta_{5 i} \text { Balcom }+\beta_{6 i} \text { IPCA }+\beta_{7 i} \text { PTAX }
$$

Os resultados das regressões são apresentados na tabela $1^{1}$, onde é possível verificar a especificidade de cada empresa, o que faz com que os títulos reajam de forma diferente a cada fator.

O grau de ajustamento foi analisado por intermédio do $\mathrm{R}^{2}$ ajustado e pode-se verificar que a explicação da variabilidade dos retornos está entre aproximadamente 7\% (EMBR3) e 33,36\% (RAPT4). Embora outras variáveis não incluídas no modelo possam explicar melhor os retornos, deve-se considerar sua significância estatística, pois uma vez que quanto maior for o número de variáveis maior será o grau de ajustamento. Isso pode ser de pouca relevância, pois as variáveis adicionadas podem não apresentar nenhuma significância estatística e econômica.

\footnotetext{
${ }^{1}$ Os resultados do teste ADF para todos os resíduos das equações estimadas por mínimos quadrados ordinários mostraram ser possível rejeitar a hipótese nula de não estacionariedade. Logo, conforme Gujarati (2006), é possível afirmar que as regressões são cointegrantes e que os coeficientes betas são parâmetros cointegrantes, podendo ser analisados.
}

O índice Ibovespa é o principal determinante nos retornos das ações e foi significativo para todas as empresas listadas, exceto para o ativo EMBR3 (Embraer) que não se mostrou sensível ao índice. Este ativo demonstrou uma relação positiva com o índice Dow Jones, o qual também pode ser considerado como proxy do mercado externo. Deve ser considerado, neste caso, o amplo crescimento da empresa em relação ao setor externo, pois ela mantém representantes em mais de oitenta países nos cinco continentes.

Além disso, este resultado é compatível com aqueles encontrados por Vechia (2008), que analisou as empresas componentes do índice IBRX-50 utilizando modelo de regressões múltiplas e também identificou que o ativo representativo da Embraer demonstrava insensibilidade ao índice de mercado brasileiro. 


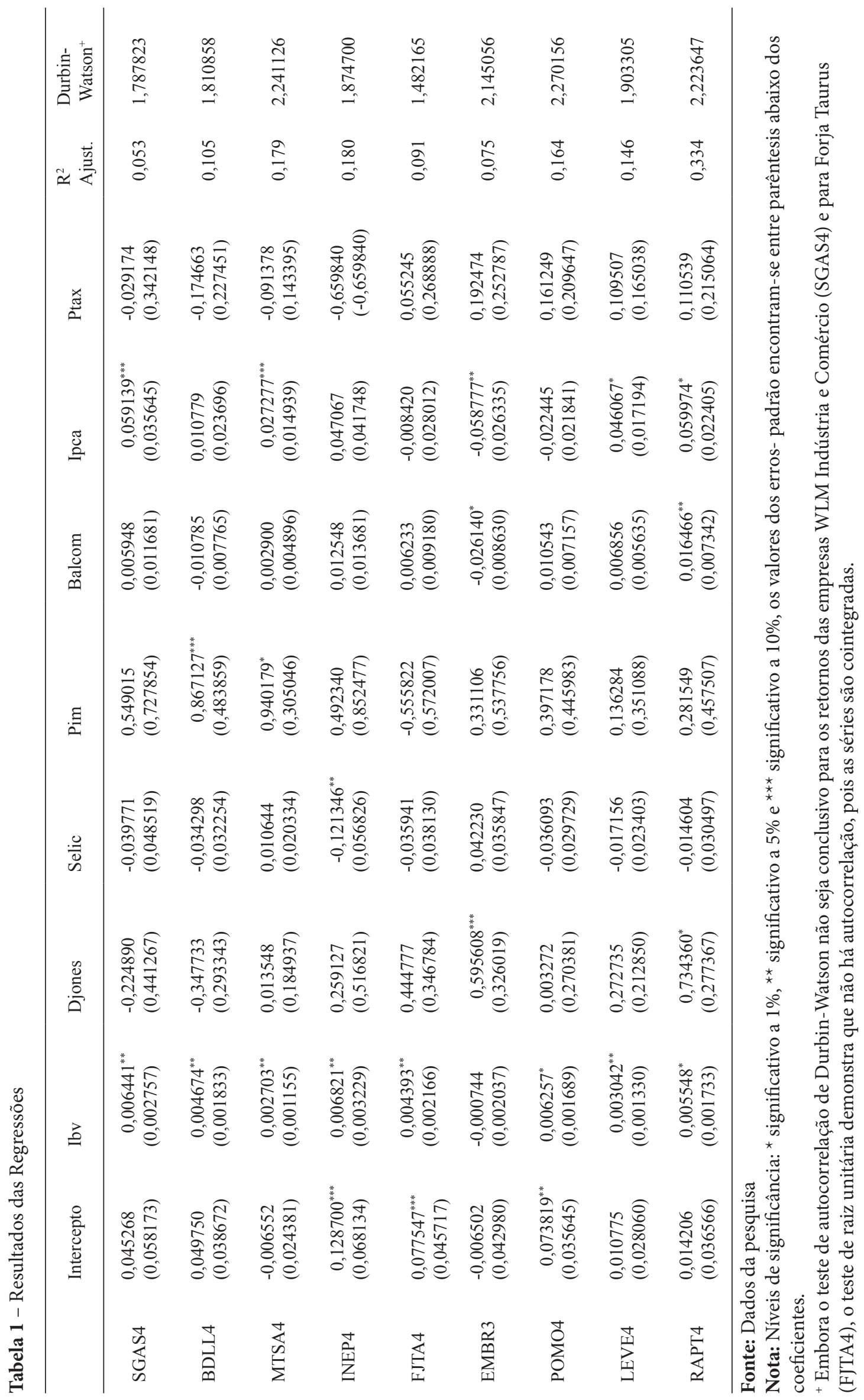


Ainda em relação ao índice americano, com exceção da Embraer, ele foi significativo apenas para a ação RAPT4 (Random S.A). A variável SELIC se mostrou significativa apenas para o retorno do ativo da Inepar S.A. (INEP4). A balança comercial, por sua vez, apenas foi significativa para a Embraer e para a Random.

A produção industrial foi estatisticamente significativa apenas para os retornos das Empresas Metisa (MTSA4) e Bardella (BDLL4), indicando uma relação positiva entre os retornos e a variável. A taxa de inflação entre as nove empresas foi significativa para cinco delas, indicando uma relação positiva para as empresas WLM Indústria e Comércio, com 10\% de significância, Metisa S.A (MTSA4), também a $10 \%$ de significância, Mahle-Metal Leve S.A (LEVE4) e Random S.A. (RAPT4), estas a $1 \%$ de significância.

Uma importante observação em relação à taxa de inflação é a sua relação negativa entre os retornos da Embraer (EMBR3) e o fator, indicando que distúrbios na demanda e oferta interna podem afetar negativamente seus retornos quando comparados com suas atividades externas.

A variável PTAX (câmbio) não foi estatisticamente significativa para nenhuma empresa da amostra. A justificativa pode se dar ao fato de apresentar uma correlação negativa, ainda que baixa, com a variável Ibovespa.

Em resumo, é possível evidenciar que existem outros fatores que influenciam os retornos das ações. Fato relevante é que apenas para os retornos da empresa Forja Taurus (FJTA4) e da Marcopolo S.A (POMO4) somente o índice Bovespa foi estatisticamente significativo, indicando que a variável de mercado é a única fonte de risco e que sua variação é melhor explicada pelo CAPM.

Por outro lado, como as variáveis explicativas utilizadas explicaram apenas uma pequena parte dos retornos das ações e dada a dificuldade levantada pelo fato de que as variáveis não são significativas de forma homogênea para o conjunto de ações, pode-se alternativamente prever o comportamento das ações quando ocorrem choques aleatórios nas variáveis macroeconômicas.

Para tanto, é possível, por meio da análise da decomposição da variância, obter informações relevantes a cada inovação sobre as variáveis que com- põem o modelo. Por esse método pode-se identificar a proporção da variância total dos retornos das ações que pode ser explicado por um choque nessas variáveis.

Entretanto, para realizar a análise de decomposição da variância, é necessário verificar a existência de causalidade entre as variáveis. Embora importante, esse teste foi efetuado apenas para definir a ordem em que as variáveis seriam dispostas na equação da análise de decomposição, visto as diferenças de respostas dos retornos as variáveis.

O Teste de Causalidade de Granger, de modo geral, analisa se os valores defasados são preditores significativos de outra série e se a relação inversa se verifica ou não de forma significativa. Ambos os testes foram calculados pelo software Eviews 5, cujos resultados levaram em conta uma defasagem e um nível de significância de rejeição da hipótese nula $(H o)$ de $10 \%$, que indica que uma variável $(\mathrm{X})$ não causa no sentido de Granger a variável (Y). Este teste demonstra apenas a precedência temporal e não causa e efeito.

Portanto, a ordem em que as variáveis se apresentam nas análises de decomposição da variância, apresentadas a seguir (tabelas 2 a 10), foi definida de acordo com os critérios de causalidade no sentido de Granger, e os resultados avaliam o poder de explicação em intervalos mensais de tempo, com os desequilíbrios de curto prazo corrigidos pelo mecanismo de correção de erro. 
Tabela 2 - Decomposição da variância SGAS4

\begin{tabular}{|c|c|c|c|c|c|c|c|c|c|}
\hline Período & S,E, & SGAS4 & IBV & DJONES & PIM & BALCOM & SELIC & PTAX & IPCA \\
\hline 1 & 0,204919 & 100,0000 & 0,000000 & 0,000000 & 0,000000 & 0,000000 & 0,000000 & 0,000000 & 0,000000 \\
\hline 2 & 0,246491 & 94,84897 & 1,397107 & 0,000820 & 0,121694 & 1,210509 & 0,007274 & 0,217560 & 2,196062 \\
\hline 24 & 0,760609 & 94,78504 & 1,091303 & 0,038825 & 0,456216 & 1,685615 & 0,031812 & 0,093375 & 1,817813 \\
\hline
\end{tabular}

Fonte: Dados da pesquisa

Tabela 3 - Decomposição da variância BDLL4

\begin{tabular}{|c|c|c|c|c|c|c|c|c|c|}
\hline Periodo & S,E, & BDLL4 & PIM & DJONES & IBV & SELIC & BALCOM & PTAX & IPCA \\
\hline 1 & 0,130673 & 100,0000 & 0,000000 & 0,000000 & 0,000000 & 0,000000 & 0,000000 & 0,000000 & 0,000000 \\
\hline 2 & 0,156304 & 94,75737 & 0,440743 & 0,678130 & 0,383107 & 0,457989 & 0,036266 & 2,989065 & 0,257328 \\
\hline 24 & 0,468511 & 91,58626 & 2,049382 & 1,510124 & 0,133113 & 0,325346 & 0,578926 & 3,595581 & 0,221269 \\
\hline
\end{tabular}

Fonte: Dados da pesquisa

Tabela 4 - Decomposição da variância MTSA4

\begin{tabular}{|c|c|c|c|c|c|c|c|c|c|}
\hline Período & S,E, & MTSA4 & PIM & DJONES & IBV & BALCOM & SELIC & IPCA & PTAX \\
\hline 1 & 0,084615 & 100,0000 & 0,000000 & 0,000000 & 0,000000 & 0,000000 & 0,000000 & 0,000000 & 0,000000 \\
\hline 2 & 0,104778 & 84,82063 & 0,139011 & 2,252416 & 6,906589 & 1,667447 & 0,021663 & 0,374453 & 3,817793 \\
\hline 24 & 0,291123 & 88,19179 & 0,179546 & 1,319501 & 4,774153 & 1,804923 & 0,005856 & 0,820528 & 2,903704 \\
\hline
\end{tabular}

Fonte: Dados da pesquisa

Tabela 5 - Decomposição da variância INEP4

\begin{tabular}{|c|c|c|c|c|c|c|c|c|c|}
\hline Período & S,E, & INEP4 & DJONES & PIM & IBV & SELIC & BALCOM & PTAX & IPCA \\
\hline 1 & 0,208791 & 100,0000 & 0,000000 & 0,000000 & 0,000000 & 0,000000 & 0,000000 & 0,000000 & 0,000000 \\
\hline 2 & 0,215661 & 95,51760 & 1,065564 & 0,332225 & 2,354944 & 0,395599 & 0,060380 & 0,160577 & 0,113110 \\
\hline 24 & 0,219038 & 92,65867 & 1,099208 & 0,374361 & 2,590624 & 2,220678 & 0,344137 & 0,216176 & 0,496144 \\
\hline
\end{tabular}

Fonte: Dados da pesquisa

Tabela 6 - Decomposição da variância FJTA4

\begin{tabular}{|c|c|c|c|c|c|c|c|c|c|}
\hline Período & $\mathrm{S}, \mathrm{E}$, & FJTA4 & PIM & IBV & DJONES & SELIC & BALCOM & PTAX & IPCA \\
\hline 1 & 0,148135 & 100,0000 & 0,000000 & 0,000000 & 0,000000 & 0,000000 & 0,000000 & 0,000000 & 0,000000 \\
\hline 2 & 0,173482 & 97,97489 & 0,291352 & 0,814209 & 0,180549 & 0,009477 & 0,236455 & 0,296034 & 0,197039 \\
\hline 24 & 0,516778 & 96,88324 & 0,208608 & 1,006723 & 0,717178 & 0,036785 & 0,760111 & 0,291196 & 0,096163 \\
\hline
\end{tabular}

Fonte: Dados da pesquisa

Tabela 7 - Decomposição da variância FJTA4

\begin{tabular}{|c|c|c|c|c|c|c|c|c|c|}
\hline Período & S,E, & EMBR3 & DJONES & IBV & PIM & PTAX & BALCOM & SELIC & IPCA \\
\hline 1 & 0,140083 & 100,0000 & 0,000000 & 0,000000 & 0,000000 & 0,000000 & 0,000000 & 0,000000 & 0,000000 \\
\hline 2 & 0,169751 & 81,21640 & 5,928166 & 0,273202 & 4,249845 & 7,452503 & 0,821831 & 0,040198 & 0,017853 \\
\hline 24 & 0,459171 & 82,17131 & 5,449675 & 0,261863 & 5,549624 & 6,148082 & 0,277423 & 0,046460 & 0,095558 \\
\hline
\end{tabular}

Fonte: Dados da pesquisa

Tabela 8 - Decomposição da variância POMO4

\begin{tabular}{|c|c|c|c|c|c|c|c|c|c|}
\hline Período & S,E, & POMO4 & DJONES & PIM & PTAX & IBV & SELIC & BALCOM & IPCA \\
\hline 1 & 0,110263 & 100,0000 & 0,000000 & 0,000000 & 0,000000 & 0,000000 & 0,000000 & 0,000000 & 0,000000 \\
\hline 2 & 0,121301 & 94,01440 & 1,142140 & 0,028513 & 2,864873 & 0,017935 & 0,007028 & 0,002070 & 1,923043 \\
\hline 24 & 0,285142 & 93,08621 & 0,676010 & 0,872596 & 1,866891 & 0,201251 & 0,874441 & 0,098174 & 2,324430 \\
\hline
\end{tabular}

Fonte: Dados da pesquisa

Tabela 9 - Decomposição da variância LEVE4:

\begin{tabular}{|c|c|c|c|c|c|c|c|c|c|}
\hline Período & $\mathrm{S}, \mathrm{E}$, & LEVE4 & PIM & DJONES & PTAX & IBV & SELIC & BALCOM & IPCA \\
\hline 1 & 0,098760 & 100,0000 & 0,000000 & 0,000000 & 0,000000 & 0,000000 & 0,000000 & 0,000000 & 0,000000 \\
\hline 2 & 0,112951 & 98,06374 & 0,042255 & 0,754502 & 0,151204 & 0,050249 & 0,023159 & 0,217505 & 0,697383 \\
\hline 24 & 0,327126 & 97,10662 & 0,372604 & 0,153012 & 0,040005 & 0,066621 & 0,018980 & 1,149443 & 1,092721 \\
\hline
\end{tabular}

Fonte: Dados da pesquisa 
Tabela 10 - Decomposição da variância da Rapt4

\begin{tabular}{|c|c|c|c|c|c|c|c|c|c|}
\hline Período & S,E, & RAPT4 & SELIC & BALCOM & IPCA & IBV & PTAX & DJONES & PIM \\
\hline 1 & 0,129752 & 100,0000 & 0,000000 & 0,000000 & 0,000000 & 0,000000 & 0,000000 & 0,000000 & 0,000000 \\
\hline 2 & 0,148999 & 88,23620 & 0,000265 & 0,659181 & 3,194474 & 2,421341 & 0,247236 & 4,925174 & 0,316133 \\
\hline 24 & 0,368701 & 87,06878 & 1,373910 & 0,976242 & 0,756289 & 1,351595 & 0,767429 & 5,655329 & 2,050423 \\
\hline
\end{tabular}

Fonte: Dados da pesquisa

Pode-se verificar que, para as ações SGAS4, BDLL4, INEP4, FJTA4, POMO4 e LEVE4, a maior parte dos desvios causados pela variância das ações é explicada pelas inovações (variações) nos próprios retornos, respondendo por mais de $90 \%$ pelo total da variância explicada.

O mesmo resultado pode ser verficado para as ações da EMBR3, MTSA4 e RAPT4, as quais, porém, demonstram uma variação maior da variância total considerando um choque aleatório no primeiro mês; como pode-se observar, o total da variância explicada pelos próprios retornos tem uma queda de aproximadamente de $12 \%$ para RAPT 4 , de $16 \%$ para MTSA4 e de $19 \%$ para a EMBR3.

Mesmo que tenham uma variação maior no primeiro mês, elas se mostram constantes ao longo de 24 meses, demonstrando uma relação de equilíbrio durante os períodos subsequentes.

Dentre as variáveis macroeconômicas utilizadas para explicação dos retornos para essas três ações, as mais relevantes foram a PTAX, Dow Jones, Ibovespa e produção industrial, de modo que a relação da PTAX com os retornos da MTSA4 e EMBR3 se dá de forma decrescente. Inicialmente ela explica aproximadamente $7,45 \%$ dos retornos da EMBR3 e 3,81\% da MTSA4, ao longo dos 24 meses o poder de explicação dessas variáveis cai para $6,14 \%$ para EMBR3 e 2,90\% para MTSA4.

Ainda para ação da MTSA4, o Ibovespa demonstra um declínio do seu poder de explicação, contribuindo inicialmente com $6,90 \%$ da variância total do retorno e com $4,77 \%$ após os períodos projetados.

O Índice Dow Jones, quando utilizado para explicar os retornos da ação EMBR3, demonstra alternância de aumento e quedas de seus valores, porém ao término do período uma inovação no índice explica 5,44\% da variância dos retornos da ação. Já para ação da RAPT4, o poder de explicação do índice se mostra de forma crescente, sendo um choque inicial na variável; seu poder de explicação da variância é de $4,92 \%$, ao passar dos meses e vai aumentando até chegar em $5,65 \%$ do total da variância explicada.
Por fim, a produção industrial apresenta-se como variável importante para as ações da EMBR3 e da RAPT4, com uma crescente contribuição para explicação da variância total desses retornos, partindo de 4,24\% para ação da EMBR3 e de 0,31\% da ação da RAPT4, chegando a 5,54\% da EMBR3, e $2,05 \%$ da RAPT4.

\section{Considerações finais}

O objetivo geral deste trabalho foi verificar se determinados fatores econômicos exercem alguma influência sobre os retornos das ações do setor de bens industriais negociadas na Bovespa no período de dezembro de 1999 a junho de 2010.

Por razões metodológicas, foi possível analisar o comportamento do retorno de nove empresas de um total de trinta e seis empresas do setor de bens industriais listadas na Bolsa de Valores.

Para o teste da APT foram utilizados os retornos mensais das nove empresas, e considerados a produção industrial, taxa de inflação, Índice Bovespa, Índice Dow Jones, balança comercial, taxa de câmbio e taxa SELIC como fatores macroeconômicos, os quais foram definidos por meio do teste de correlação a fim de evitar a colinearidade dos fatores.

Considerando-se o tamanho da amostra e a quantidade de variáveis, fez-se necessário analisar a estacionariedade dos dados para evitar o fenômeno de resultados irrealistas ou espúrios. Como resultado, somente a taxa SELIC não se mostrou estacionária para o período analisado. No entanto, os resíduos das regressões realizadas com esta variável são estacionários, o que indica que as variáveis são cointegradas e que os resultados não são espúrios.

Os retornos das ações não são determinados pelos mesmos fatores nem são influenciados por eles na mesma magnitude. Verificou-se que, além do mercado (Ibovespa) que foi não significativo apenas para os retornos da Embraer, a taxa de inflação foi significativa para cinco das nove empresas analisadas. 
A taxa de câmbio, por sua vez, não foi significativa para nenhuma das equações. Isto pode ser explicado pelo fato de que a grande maioria das empresas não tem no mercado externo o foco de sua atuação, e aquelas que operam globalmente, caso da Embraer e da Random, não experimentam flutuações na demanda por conta do câmbio.

Embora os resultados evidenciem que as variáveis explicativas utilizadas nas regressões exercem pequena influência sobre os retornos das ações do setor de bens industriais, ainda assim é possível corroborar o modelo da APT, o qual afirma que outras fontes de risco, além do mercado, influenciam os retornos das ações.

Os resultados obtidos pela análise de regressão puderam ser confirmados pelo teste de decomposição da variância, o qual demonstrou que a maior parte dos desvios causados pela variação dos retornos é explicada pelas inovações nos próprios retornos.

Os resultados obtidos para as ações da EMBR3, RAPT4 e MTSA4 evidenciaram que choques aleatórios em um primeiro momento causam uma queda maior da variância total explicada pela variância dos próprios retornos, porém respondem com equilíbrio aos meses subsequentes. A principal contribuição do teste se deve ao fato de observar-se que variáveis utilizadas contribuem para explicar os retornos das ações.

A variável PTAX, apesar de se mostrar significativa ao longo do período, teve uma contribuição decrescente para o total da variância explicada pela variável.

Finalmente, embora outras técnicas mais avançadas possam ser utilizadas para tentar prever o retorno das ações, foi possível observar a influência individual de cada variável sobre um conjunto de ações. Isso possibilita ao investidor assumir diversas posturas quanto a vários fatores de risco. Ele pode optar em construir uma carteira que acompanhe esses fatores de modo mais próximo, ou, através das variáveis significativas, apostar nas variações futuras desses fatores, construindo carteiras que lhe forneçam retornos esperados superiores ao mercado.

\section{Referências}

BEENSTOCK, M. ;CHAN, K. F. Economic Forces in the London Stock Market. Oxford Bulletin of Economics and Statistics v. 50, n. 1, p. 27-39, 1988
BURMEISTER, E., ROLL, R., ROSS, S. Using macroeconomic factors to control portfolio risk. BIRR Working Paper, 2003. Disponível em: <http://www.birr. com/Using_Macroeconomic_Factors.pdf $>$. Acessado em 19/5/2011.

CALLADO, A. A. C. et.al. Relação entre Retornos das Ações e Variáveis Macroeconômicas: Um Estudo entre Empresas do Setor de Alimentos e Bebidas Através do Modelo da APT. In: Congresso Sociedade Brasileira de Economia, Administração e Sociologia Rural, 47., 2009, Porto Alegre. Apresentação oral- economia e gestão no agronegócio. Disponível em: <http://www.sober.org.br/palestra/13/1037. pdf $>$. Acesso em mar. 2010.

GUJARATI, D. N. Econometria básica. Rio de Janeiro: Elsevier, 2006. 812p.

ROSS, Stephen A. The Arbitrage Theory of Capital Asset Pricing, Journal of Economic Theory, v. 13, p.341-360, 1976.

ROSTANGO, L., KLOECKNER, G. O., BECKER, J. L. Previsibilidade de retornos das Ações na Bovespa: Um Teste Envolvendo o Modelo de Fator de Retorno Esperado. Brazilian Finance Review. v.2, n.2, p. 183- 206. São Paulo, 2004.

VECHIA, D. D. Análise de fatores econômicos no desempenho de ações na Bovespa. 2008, 53 f. Trabalho de Conclusão de Curso (Bacharel em Administração)Universidade Federal do Rio Grande do Sul, Rio Grande do Sul, 2008.
Recebido em: 13 de outubro de 2011 Aceito em: 17 de setembro de 2012 